\title{
SMOOTH OPTIMAL SYNTHESIS FOR INFINITE HORIZON VARIATIONAL PROBLEMS
}

\author{
Andrei A. Agrachev ${ }^{1}$ and Francesca C. Chittaro ${ }^{2}$
}

\begin{abstract}
We study Hamiltonian systems which generate extremal flows of regular variational problems on smooth manifolds and demonstrate that negativity of the generalized curvature of such a system implies the existence of a global smooth optimal synthesis for the infinite horizon problem. We also show that in the Euclidean case negativity of the generalized curvature is a consequence of the convexity of the Lagrangian with respect to the pair of arguments. Finally, we give a generic classification for 1-dimensional problems.
\end{abstract}

Mathematics Subject Classification. 93B50, 49K99.

Received July 30, 2007.

Published online April 26, 2008.

\section{IntroduCtion}

Given a smooth $n$-dimensional manifold $M$ and a smooth function $\varphi: T M \rightarrow \mathbb{R}$ we study the functional

$$
J(\gamma(\cdot))=\int_{0}^{\infty} \varphi(\gamma(t), \dot{\gamma}(t)) \mathrm{d} t
$$

defined on the Lipschitzian curves $\gamma:[0,+\infty) \rightarrow M$ such that the integral (1) converges. More precisely, we assume that an equilibrium point $q_{\infty} \in M$ is fixed such that $\varphi\left(q_{\infty}, 0\right)=0, \frac{\partial \varphi}{\partial q}\left(q_{\infty}, 0\right)=0$, and try to find the cost

$$
c\left(q_{0}\right)=\min \left\{\int_{0}^{\infty} \varphi(\gamma(t), \dot{\gamma}(t)) \mathrm{d} t: \quad \gamma(0)=q_{0}, \lim _{t \rightarrow \infty} \gamma(t)=q_{\infty}\right\} .
$$

Moreover, we are mainly interested in the characterization of Lagrangians $\varphi$ such that the minimization problem admits a smooth optimal synthesis according to the following definition:

\footnotetext{
Keywords and phrases. Infinite-horizon, optimal synthesis, Hamiltonian dynamics.

1 SISSA, via Beirut 2-4, 34014 Trieste, Italy. agrachev@sissa.it

2 Dipartimento di Matematica Applicata "G. Sansone", via S. Marta 3, 50139 Firenze, Italy. chittaro@math.unifi.it
} 
Definition 1.1. A smooth optimal synthesis is a smooth vector field $V$ on $M$ such that $q_{\infty}$ is a globally stable equilibrium of the ordinary differential equation $\dot{q}=V(q)$ and

$$
c(q)=\int_{0}^{\infty} \varphi\left(\gamma_{q}(t), \dot{\gamma}_{q}(t)\right) \mathrm{d} t, \quad \forall q \in M,
$$

where $\dot{\gamma}_{q}(t)=V\left(\gamma_{q}(t)\right), \gamma_{q}(0)=q$.

The classical case of quadratic Lagrangians:

$$
\varphi(q, \dot{q})=\langle R \dot{q}, \dot{q}\rangle+\langle S q, q\rangle, \quad q \in \mathbb{R}^{n}, \quad q_{\infty}=0,
$$

demonstrates that the problem is somehow natural. Indeed, it is easy to show that problem (1.3) admits a smooth optimal synthesis (with a linear field $V$ ) if the matrices $R$ and $S$ define positive definite quadratic forms. On the other hand, if one of these quadratic forms is sign-indefinite, then $c(q)$ is simply not defined for almost all $q \in \mathbb{R}^{n}$.

Of course, smooth optimal synthesis may exist only on the contractible (i.e. homeomorphic to $\mathbb{R}^{n}$ ) manifold. Nevertheless, we prefer to use intrinsic coordinate free language. The reason is clear: any smooth change of variables transforms optimal synthesis into optimal synthesis, hence natural conditions for the existence of a smooth optimal synthesis have to survive smooth changes of variables, in other words, have to be coordinates free.

In fact, the manifold $M$ often appears as a domain in a bigger manifold or as the universal covering of a hyperbolic Riemannian manifold. In particular, if the Hessian of $\varphi$ at $\left(q_{\infty}, 0\right)$ is positive definite, then the restriction of our problem to a small neighborhood of $q_{\infty}$ admits a smooth optimal synthesis which is a small perturbation of the synthesis for the corresponding quadratic problem. The main goal of this paper is to find natural intrinsic conditions which guarantee the existence of a global smooth optimal synthesis for given $M$.

Any segment of a minimizing path $\gamma(t), t \geq 0$, is automatically a minimizer for the corresponding finite horizon functional. The extremal paths of the finite horizon problems are described either by the EulerLagrange second order ordinary differential equation on $M$ or by the Hamiltonian system on $T^{*} M$. In the classical Calculus of Variations, usually the former approach is preferred (see for instance the textbooks $[5,6]$ ); we use the Hamiltonian approach, in the spirit of the Pontryagin Maximum Principle.

Clearly, smooth optimal synthesis should correspond to an $n$-dimensional stable invariant submanifold of the Hamiltonian system. Hence we need conditions which guarantee the existence of an appropriate global invariant submanifold. Moreover, we would like to have conditions which are reduced to the sign conditions for the quadratic forms defined by the matrices $R$ and $S$ in the case of the quadratic Lagrangian (1.3). To this end, we use curvature-type invariants of the Hamiltonian systems. Negativity of the curvature plus some standard growth conditions not only implies the existence of an $n$-dimensional stable submanifold of the Hamiltonian system in $T^{*} M$ but also guarantees that this submanifold is diffeomorphically projected onto $M$.

Section 2 contains necessary facts on Hamiltonian systems. The main results are stated and proved in Section 3. Section 4 is mainly devoted to the case of convex Lagrangians in the Euclidean space. The last Section 5 contains a generic classification for 1-dimensional problems: a preliminary step towards a more flexible theory which would allow the curvature to change sign.

The literature devoted to the infinite horizon optimization is vast and we do not plan to observe it here. Let us only mention a very recent paper [4] on the infinite horizon optimal synthesis in a very general fairly nonsmooth and even discontinuous setting. 


\section{Statement of the PRoblem AND PRELiminaries}

\subsection{The problem}

Let $M$ be a complete Riemannian $n$-dimensional manifold; we consider problem (1.1), and let us formulate it as an optimal control problem:

$$
\min _{q(t)} \int_{0}^{\infty} \varphi(q(t), u(t)) \mathrm{d} t
$$

where $q:[0,+\infty) \rightarrow M$ is a Lipschitzian curve such that

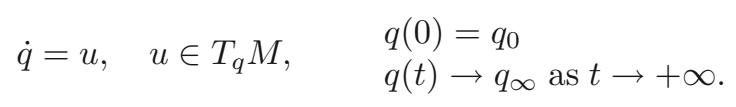

We make the following assumptions:

(H1) $\varphi$ is bounded from below and is strongly convex w.r.t. the second variable; moreover, we assume that $\varphi$ grows superlinearly in the second variable with respect to the given Riemannian metric, i.e. $\varphi(q, u)+c>0$ for some constant $c$ and

$$
\frac{|u|}{\varphi(q, u)+c} \rightarrow 0 \quad \text { as }|u| \rightarrow+\infty
$$

(H2) there is a unique point $q_{\infty} \in M$ such that $\varphi\left(q_{\infty}, 0\right)=0$ and $\frac{\partial \varphi}{\partial q}\left(q_{\infty}, 0\right)=0$;

(H3) there exist constants $a, b>0$ such that for any $(q, u)$

$$
\left|\partial_{q} \varphi(q, u)\right| \leq a(\varphi(q, u)+|u|)+b,
$$

where $\partial_{q}$ is the covariant derivative.

Let us recall that the cotangent bundle $T^{*} M$ admits a natural symplectic structure, given by the symplectic form $\sigma$, which is defined as $\sigma=\mathrm{d} \vartheta$, where for any $\lambda \in T^{*} M \vartheta_{\lambda}:=\lambda \circ \pi_{*}$, and $\pi: T^{*} M \rightarrow M$ is the canonical projection. A subspace of the tangent space to $T^{*} M$ is called Lagrangian if the symplectic form vanishes on it and if it has the maximal possible dimension compatible with this property, which is $\operatorname{dim}\left(T^{*} M\right) / 2$ (in this case, $n$ ); the set of all Lagrangian subspaces of a vector space is called Lagrange Grassmannian of the vector space. A submanifold of some symplectic manifold is called Lagrangian if its tangent space at every point is a Lagrangian subspace. Moreover, if $h: T^{*} M \rightarrow \mathbb{R}$ is a smooth function on the cotangent bundle, we can define the Hamiltonian vector field $\vec{h}$ associated to $h$ through the following relation $\sigma_{z}(\vec{h}, \cdot)=-\mathrm{d}_{z} h$, where $\mathrm{d}_{z} h$ is the differential of the function $h$ at $z \in T^{*} M$.

Let us recall moreover that an admissible control is a measurable locally bounded mapping $u: t \mapsto u(t) \in T M$; a pair $(q(\cdot), u(\cdot))$ such that $\dot{q}=u$ is called admissible pair. It is well known (see for instance the text book [3]) that we can associate to problem (2.1) a Hamiltonian function $h_{u}(\lambda)=\langle\lambda, u\rangle-\varphi(q, u)$, where $\lambda \in T^{*} M$, and Pontryagin Maximum Principle states that if $\tilde{u}(t)$ is an admissible control and $\tilde{q}(t)$ the corresponding solution of Cauchy problem (2.2), then $\tilde{q}(t)$ is an optimal trajectory for (2.1) only if there exists a Lipschitzian curve $\lambda(t) \in T_{\tilde{q}(t)}^{*} M, 0 \leq t<+\infty$, such that

$$
\begin{aligned}
\dot{\lambda}(t)= & \vec{h}_{\tilde{u}(t)}(\lambda(t)) \\
h_{\tilde{u}(t)}(\lambda(t))= & \max _{u \in T_{q} M} h_{u}(\lambda(t)) \\
& \text { for almost all } t \in[0,+\infty) ;
\end{aligned}
$$

we have that $\pi(\lambda(t))=\tilde{q}(t)$ for $t \in[0,+\infty)$. 


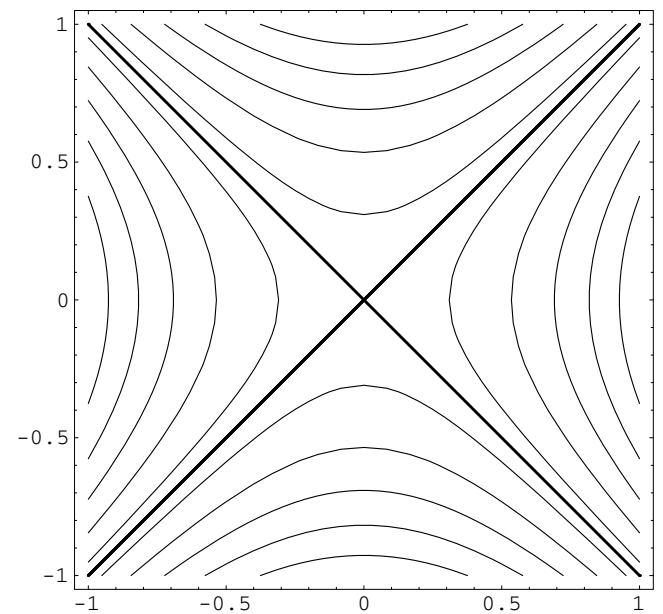

Figure 1. Optimal synthesis for the inverted harmonic oscillator.

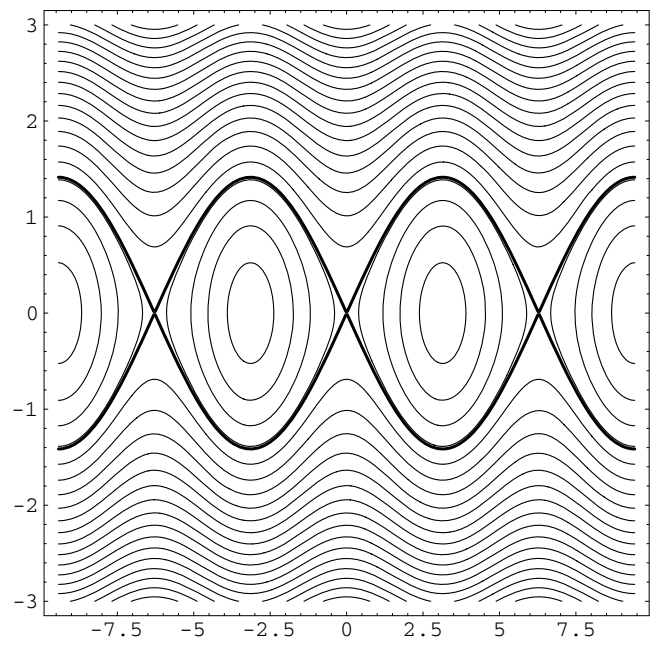

Figure 2. Optimal synthesis for the pendulum.

We define the maximized Hamiltonian

$$
H(\lambda)=\max _{u \in T_{q} M} h_{u}(\lambda)
$$

Assumptions (H1) and (H3) imply that the Hamiltonian $H$ is smooth and the Hamiltonian field $\vec{H}$ is complete.

Actually, in the following we will only need that the Hamiltonian satisfies these two assumptions (smoothness and completeness of the vector field); hypotheses (H1) and (H3) are thus assumed to be satisfied in order to guarantee the required properties.

The vector field $\vec{H}$ defines the Hamiltonian dynamical system on $T^{*} M$ given by the equation

$$
\dot{\lambda}(t)=\vec{H}(\lambda(t))
$$

we denote with $\phi^{t}$ the flow generated by the vector field $\vec{H}$ through the equation (2.5). In the following, we will say that a trajectory of this system is bounded if it has compact closure. Hypothesis (H2) implies that there exist a point $z_{\infty} \in T_{q_{\infty}}^{*} M$ which is an equilibrium of the system (2.5).

Since the maximized Hamiltonian $H$ is smooth, it is known that if a Lipschitzian curve $\lambda(t) \in T^{*} M$ is a solution of the Hamiltonian system (2.5), then one can choose and admissible control $\tilde{u}(t), t \in[0,+\infty)$, such that the pair $(\lambda(t), \tilde{u}(t))$ satisfies equations $(2.3)-(2.4)$ (see [3]).

The aim of this paper is to find the condition for the existence of a smooth optimal synthesis for the problem (2.1) focusing on the dynamical properties of the Hamiltonian system (2.5).

Before presenting the technical tools, let us see what happens in two elementary examples with $M=\mathbb{R}$, $q_{\infty}=0$, given by $\varphi(q, u)=\frac{1}{2} u^{2}+\frac{1}{2} q^{2}, H(p, q)=\frac{1}{2} p^{2}-\frac{1}{2} q^{2}$ (the inverted harmonic oscillator) and $\varphi(q, u)=$ $\frac{1}{2} u^{2}+1-\cos (q), H(p, q)=\frac{1}{2} p^{2}-1+\cos (q)$ (the pendulum). Here $T^{*} \mathbb{R}$ is identified with $\mathbb{R}^{2}$, so that $\lambda=(p, q) \in$ $T_{q}^{*} \mathbb{R}$; the phase diagrams of these Hamiltonian systems are depicted respectively in Figures 1 and 2.

In the first case, every trajectory is unstable (i.e. goes to infinity) but the ones whose initial points lie on the bisectrix of the II and the IV quadrant: every semitrajectory arising from these points reaches the origin in an infinite time; since the projection of the bisectrix on the state axis is a bijection, we can guess that for any initial $q$ there is a Hamiltonian trajectory that goes to the origin. It is easy to show that actually this trajectory is the optimal solution of our problem. 
In the second case, we shall distinguish different possibilities. The Hamiltonian trajectories lying on energy levels with $H>0$ are all unstable; for $H<0$ the trajectories are closed and do no reach the origin; but for any initial $q_{0} \in(-2 \pi, 2 \pi)$ there is a trajectory that lies on the level $H=0$ and that reaches the origin in an infinite time, and is an optimal trajectory for the problem under investigation. If $\left|q_{0}\right|>2 \pi$, the trajectories on the zero level arising form $q_{0}$ do not reach the origin, but go to another fixed point of the system, and then cannot be optimal.

These examples suggest us that the we shall look for the optimal trajectories among the stable trajectories of the Hamiltonian system (2.5).

\subsection{Geometric tools}

Here we briefly explain the formalism introduced in $[1,2]$ that permits us to recover a lot of information about the structure of our Hamiltonian system.

Let $t \mapsto \Lambda(t)$ be a smooth curve in the Lagrange Grassmannian of $T_{z}\left(T^{*} M\right)$, and let $\frac{\mathrm{d}}{\mathrm{d} t} \Lambda(t)$ be the tangent vector to it; for any $\tau$, we can canonically associate to the tangent vector a quadratic form on $\Lambda(\tau)$ denoted by $\dot{\Lambda}_{\tau}$ and defined as $\dot{\Lambda}_{\tau}(\xi)=\sigma(\dot{\lambda}(\tau), \lambda(\tau))$, where $\lambda(t)$ is a smooth curve in $T_{z}\left(T^{*} M\right)$ such that $\lambda(\tau)=\xi$, $\lambda(t) \in \Lambda(t)$ for any $t$, and $\dot{\lambda}(t)$ is its derivative; it can be shown that actually the quadratic form depends only on $\left.\frac{\mathrm{d}}{\mathrm{d} t} \Lambda(t)\right|_{t=\tau}$. We say that the curve $\Lambda(t)$ is regular if the quadratic form $\dot{\Lambda}_{t}$ is nondegenerate, and that the curve is monotone increasing (decreasing) if $\dot{\Lambda}_{t}$ is positive (negative) definite. To a quadratic form on some vector space it is uniquely associated a self-adjoint linear operator from the vector space to its dual; then, we can associate to the quadratic form $\dot{\Lambda}_{t}$ a linear operator $\bar{\Lambda}_{t}: \Lambda(t) \rightarrow \Lambda(t)^{*}$.

A Lagrangian distribution is a smooth family $\left\{\Lambda_{z}\right\}_{z \in T^{*} M}$ of Lagrangian subspaces, where $\Lambda_{z} \subset T_{z}\left(T^{*} M\right)$. Given $z \in T^{*} M$ we define a curve $\Lambda_{z}(t):=\phi^{-t}{ }_{*} \Lambda_{\phi^{t}(z)}$ in the Lagrange Grassmannian of $T_{z}\left(T^{*} M\right)$. We have $\dot{\Lambda}_{z}(\xi)=\sigma([\vec{H}, X](z), X(z))$ for any $\xi \in T_{z}\left(T^{*} M\right)$ and any vector field which is a section of our distribution such that $X(z)=\xi$.

Remark 2.1. Let $\Lambda_{z}=T_{z}\left(T_{\pi(z)}^{*} M\right)$, and choose on $T^{*} M$ Darboux coordinates $\{(p, q)\}$ in such a way that $T_{q}^{*} M=\left\{(p, q): p \in \mathbb{R}^{n}\right\}$; then $\dot{\Lambda}$ is represented in these coordinates by the Hessian matrix $\frac{\partial^{2} H}{\partial p^{2}}$, and then monotonicity of the curve $\Lambda_{z}(t)$ is equivalent to convexity or concavity of $H$ on the fibers of the cotangent bundle.

Now assume that $\left\{\Pi_{z}\right\}_{z \in T^{*} M}$ is another Lagrangian distribution on $T^{*} M$, and assume that $\Lambda_{z}$ and $\Pi_{z}$ are transversal at any point (i.e. $\Lambda_{z} \cap \Pi_{z}=0$ ), that means that they define for any point a splitting of the tangent space to $T^{*} M$. The symplectic form $\sigma$ defines a nondegenerate pairing of $\Lambda_{z}$ and $\Pi_{z}$ as well as $\Lambda_{z}(t)$ and $\Pi_{z}(t)$, where $\Pi_{z}(t)=\phi^{-t}{ }_{*} \Pi_{\phi^{t}(z)}$. In particular, $\Lambda_{z}(t)^{*}$ is identified with $\Pi_{z}(t)$ and $\Pi_{z}(t)^{*}$ is identified with $\Lambda_{z}(t)$, and we may treat the operator $\overline{\dot{\Lambda}}_{z_{t}}$ as a linear mapping from $\Lambda_{z}(t)$ to $\Pi_{z}(t)$, and the operator $\bar{\Pi}_{z_{t}}$ as a linear mapping from $\Pi_{z}(t)$ to $\Lambda_{z}(t)$.

Assume that $\Lambda_{z}(t)$ is regular and monotone; the following rules uniquely define a Riemannian structure $\langle\cdot, \cdot\rangle$ on $T^{*} M$ associated with the Hamiltonian $H$ and the splitting $T\left(T^{*} M\right)=\Lambda \oplus \Pi$ :

$$
\langle\xi, \xi\rangle=\left|\dot{\Lambda}_{z_{0}}(\xi)\right|,\langle\eta, \eta\rangle=\left|\dot{\Lambda}_{z_{0}}\left(\left(\overline{\dot{\Lambda}_{z_{0}}}\right)^{-1} \eta\right)\right|,\langle\xi, \eta\rangle=0,
$$

for all $\xi \in \Lambda_{z}, \eta \in \Pi_{z}, z \in T^{*} M$.

Another important object associated with the Lagrangian splitting is the operator $R_{z}^{H}: \Lambda_{z} \rightarrow \Lambda_{z}$ defined as follows:

$$
R_{z}^{H}=-\overline{\dot{\Pi}_{z_{0}}} \circ \overline{\dot{\Lambda}_{z_{0}}}
$$

The operator (9) is called the generalized curvature operator of the Hamiltonian vector field $\vec{H}$ at the point $z$ with respect to the splitting $\Lambda \oplus \Pi$.

If $\Lambda_{z}(t)$ is regular and monotone, then the generalized curvature is a self-adjoint operator with respect to the scalar product on $\Lambda_{z}$ defined above [1]; in particular, it has real eigenvalues. 
Any vector field $Y$ on $T^{*} M$ is splitted $Y=Y_{\Lambda}+Y_{\Pi}$, where $Y_{\Lambda}$ is a section of $\Lambda$ and $Y_{\Pi}$ is a section of $\Pi$. For any section $X$ of $\Lambda$ and any $z \in T^{*} M$, we have (see [1]):

$$
R_{z}^{H} X(z)=-\left[\vec{H},[\vec{H}, X]_{\Pi}\right]_{\Lambda}(z) .
$$

In what follows we always assume that $\Lambda_{z}=T_{z}\left(T^{*} M\right)$. Assumption (H1) implies that the curves $\Lambda_{z}(t)$ are regular and monotone. As for the complements $\Pi_{z}$, there are different ways to select them. We are now going to describe two quite natural ones: the first is canonically defined by the Hamiltonian field $\vec{H}$ and the second by a symmetric linear connection on the base manifold $M$.

Let us consider the first one. To any regular curve $\Lambda_{z}(t)$ in the Lagrange Grassmannian of $T_{z}\left(T^{*} M\right)$ we can canonically associate another smooth curve in the following way. Let $\Delta$ be a Lagrangian subspace of $T_{z}\left(T^{*} M\right)$ transversal to $\Lambda_{z}(0)$, and let $\pi_{\Delta \Lambda_{z}(0)}$ be the projector of $T^{*} M$ onto $\Lambda_{z}(0)$ and parallel to $\Delta$; then it allows that

$$
\left.\pi_{\Delta \Lambda_{z}(0)}\right|_{\Lambda_{z}(0)}=\mathrm{id} ;\left.\quad \pi_{\Delta \Lambda_{z}(0)}\right|_{\Delta}=0
$$

The space $\left\{\pi_{\Delta \Lambda_{z}(0)}: \Delta \in \Lambda_{z}(0)^{\pitchfork}\right\}$ (where $\Delta \in \Lambda_{z}(0)^{\pitchfork}$ means that $\left.\Delta \cap \Lambda_{z}(0)=0\right)$ is an affine subspace of $g l\left(T_{z}\left(T^{*} M\right)\right)$ : to prove this, notice that the linear combination

$$
\alpha \pi_{\Delta \Lambda_{z}(0)}+(1-\alpha) \pi_{\Delta \Lambda_{z}(0)} \quad \alpha \in \mathbb{R}
$$

vanishes on its kernel and equals the identity when restricted to $\Lambda_{z}(0)$, hence it is a projector $\left(\right.$ onto $\left.\Lambda_{z}(0)\right)$. On the space $\left\{\pi_{\Delta \Lambda_{z}(0)}: \Delta \in \Lambda_{z}(0)^{\pitchfork}\right\}$ it is defined the subtraction operation which gives values on $g l\left(T_{z}\left(T^{*} M\right)\right)$.

Let us now consider $\Lambda_{z}(\tau)$ and $t \neq \tau$ : since the curve is regular, $\Lambda_{z}(t)$ is transversal to $\Lambda_{z}(\tau)$ for $t$ sufficiently close to $\tau$ [1]. We say that the operator-valued function $t \mapsto \pi_{\Lambda_{z}(t) \Lambda_{z}(\tau)}$ has a pole if the function $t \mapsto$ $\pi_{\Lambda_{z}(t) \Lambda_{z}(\tau)}-\pi_{\Delta \Lambda_{z}(\tau)}$ has a pole (as a function with values in $g l\left(T_{z}\left(T^{*} M\right)\right)$ ) for some $\Delta \in \Lambda_{z}(0)^{\dagger}$. Let us compute its Laurent expansion: $\pi_{\Lambda_{z}(t) \Lambda_{z}(0)}=\pi_{0}+\sum_{i \neq 0} \pi_{i} t^{i}$. It can be proved that $\pi_{i} \in g l\left(T_{z}\left(T^{*} M\right)\right)$ for $i \neq 0$, while $\pi_{0}$ is an element of the affine subspace; then there exists a unique subspace $\Lambda_{z}^{\circ}(\tau)$ of $T_{z}\left(T^{*} M\right)$ transversal to $\Lambda_{z}(\tau)$, such that $\pi_{0}=\pi_{\Lambda_{z}(\tau) \Lambda_{z}(\tau)}$; this subspace is called the derivative element to $\Lambda_{z}(\tau)$.

This procedure can be repeated to construct the derivative element to $\Lambda_{z}(t)$ for any $t$, and hence we can define the derivative curve $t \mapsto \Lambda_{z}^{\circ}(t)$.

Since the curve $\Lambda_{z}(t)$ is regular, its derivative curve is smooth; since $\Lambda_{z}(t)$ is regular and Lagrangian, then $\Lambda_{z}^{\circ}(t)$ is Lagrangian for any $t$ (see [1] for details and explicit expressions).

The splitting $T_{z}\left(T^{*} M\right)=\Lambda_{z}(0) \oplus \Lambda_{z}^{\circ}(0)$ given by a curve and its derivative curve is called the canonical splitting.

Let us now define the second remarkable splitting. Any linear connection on $M$ defines a parallel transport of tangent vectors to $M$ along curves in $M$ and hence, by duality, a parallel transport of cotangent vectors to $M$. Hence, the connection defines a lift to $T^{*} M$ of vector fields on $M$; in other words, it defines a vector distribution on $T^{*} M$ that is a complement to the "vertical" distribution $T_{z}\left(T^{*} M\right)=\Lambda_{z}, z \in T^{*} M$. A simple calculation shows that this distribution is Lagrangian if and only if the connection is symmetric (i.e. torsion-free).

Let us now consider the Levi-Civita connection on a Riemannian manifold $(M, g)$ ( $g$ is the metric); the adjoint connection to the Levi-Civita connection then defines a Lagrangian splitting on $T\left(T^{*} M\right)$. It happens that this Lagrangian splitting coincides with the canonical splitting defined by the Hamiltonians of natural mechanical systems on the Riemannian manifold $(M, g)$. We mean Hamiltonians $H$ of the form:

$$
H(z)=\frac{1}{2}\left\langle z, g^{-1}(\pi(z)) z\right\rangle+U(\pi(z)), \quad z \in T^{*} M,
$$

where $U$ is a smooth function on $M$, a "potential energy". In this case, the generalized curvature is given by

$$
R_{z}^{H}(X)=\mathcal{R}(\bar{z}, X) \bar{z}+D_{X}(\nabla U),
$$


where $\bar{z} \in T M$ is the dual to $z$ via the metric $g$, symbol $\mathcal{R}$ denotes the Riemannian curvature, and $D$ is the covariant derivative. In the special case of $M=\mathbb{R}^{n}$ with the standard flat metric we obtain:

$$
H(p, q)=\frac{1}{2}|p|^{2}+U(q), \quad R_{z}^{H}=\frac{\partial^{2} U}{\partial q^{2}} .
$$

Let us now recall the following definition:

Definition 2.1. A point $z \in T^{*} M$ is a hyperbolic fixed point of the flow $\phi^{t}$ if it is a fixed point of the flow and if there exist a $\phi^{t}$-invariant splitting of the tangent space $T_{z}\left(T^{*} M\right)=E_{z}^{+} \oplus E_{z}^{-}$and two positive constants $\gamma$ and $b$ such that

$$
\left\|D_{z} \phi^{\mp t} X\right\| \leq b \mathrm{e}^{-\gamma t}\|X\| \text { for } X \in E_{z}^{ \pm} \text {and } t \geq 0 .
$$

Let us now mention here two results proved in [1], that show the relation between the generalized curvature and the behaviour of the Hamiltonian system; in the following two statements, we will reduce to the case in which the splitting $\Lambda \oplus \Pi$ is the canonical splitting.

Theorem 2.1. Let $\Lambda_{z}(t)$ be regular and monotone and $W$ a compact invariant set of the flow $\phi^{t}$. If $\vec{H}$ has negative curvature at any point of $W$, then $W$ is a finite set and each point of $W$ is a hyperbolic equilibrium of the field $\vec{H}$.

Corollary 2.1. Assume that $\Lambda_{z}(t)$ is regular and monotone and that $\vec{H}$ has everywhere negative curvature. Then any bounded semitrajectory of the system $\dot{z}=\vec{H}(z)$ converges to an equilibrium with exponential rate, while another semitrajectory of the same trajectory must be unbounded.

These two results can actually be generalized, using the technique that Wojtkovski proposed in [10]. The core of the proof of Theorem 2.1 and Corollary 2.1 is to find a special metric $\|\cdot\|$ on $T\left(T^{*} M\right)$ (which actually is the metric defined by $\left.\dot{\Lambda}_{z}\right)$ and a family of invariant expanding and contracting cones $C_{z}^{ \pm} \subset T_{z}\left(T^{*} M\right)$ such that

$$
\left\|D_{z} \phi^{\mp t} X\right\| \leq b \mathrm{e}^{-\gamma t}\|X\| \text { for } X \in C_{z}^{ \pm} \text {and } t \geq 0
$$

for some positive constants $\gamma, b$; to demonstrate these results it was used the negativity of the generalized curvature (with respect to the canonical splitting). In [10] it is proved that such a family of cones $C_{z}^{ \pm}$exists, provided there is a continuous nondegenerate quadratic form $\mathcal{Q}: T M \rightarrow \mathbb{R}$ such that its Lie derivative with respect to $\vec{H} \mathcal{L}_{\vec{H}} \mathcal{Q}:=\frac{\mathrm{d}}{\mathrm{d} t} \mathcal{Q}\left(D \phi^{t} \cdot\right)_{t=0}$ is positive definite. The results above stated are valid if such quadratic form exists.

In order to use the generalized versions of Theorem 2.1 and Corollary 2.1 we have then to prove the existence of such a quadratic form. The following lemma show that this requirement is satisfied under some assumptions on the sign of the curvature.

Lemma 2.1 (P. Przytycki). Let $\Lambda \oplus \Pi$ be a Lagrangian splitting of $T\left(T^{*} M\right)$ such that the curves $\Lambda_{z}(t)$ and $\Pi_{z}(t)$ are regular, and $\Lambda_{z}(t)$ is monotone. Assume that the curvature $R_{z}^{H}$ of the vector field $\vec{H}$ with respect to this splitting is negative definite for any $z$. Then there exists a continuous nondegenerate quadratic form $Q: T M \rightarrow \mathbb{R}$ such that $\mathcal{L}_{\vec{H}} \mathcal{Q}$ is positive definite.

Proof. First of all, note that asking the curvature $R_{z}^{H}$ to be negative definite is equivalent to saying that the quadratic forms associated to $\dot{\Lambda}_{z}(t)$ and $\dot{\Pi}_{z}(t)$ are both regular and have opposite sign (see [1]); for simplicity, assume that $\Lambda_{z}(t)$ is monotone increasing.

Let us write any vector $X$ on $T^{*} M$ as $X=X_{\Lambda}+X_{\Pi}$, where, as before, $X_{\Lambda}$ is a section of $\Lambda$ and $X_{\Pi}$ a section of $\Pi$; define the following quadratic form on $T M$ :

$$
\mathcal{Q}_{t}(X)=\sigma\left(\left(D_{z} \phi^{t} X\right)_{\Lambda},\left(D_{z} \phi^{t} X\right)_{\Pi}\right)
$$


we have that

$$
\begin{aligned}
\mathcal{L}_{\vec{H}} \mathcal{Q}(X)= & -\sigma\left([\vec{H}, X]_{\Lambda}, X_{\Pi}\right)-\sigma\left(X_{\Lambda},[\vec{H}, X]_{\Pi}\right) \\
= & \sigma\left(\left[\vec{H}, X_{\Lambda}\right], X_{\Lambda}\right)+\sigma\left(\left[\vec{H}, X_{\Pi}\right], X_{\Lambda}\right) \\
& +\sigma\left(X_{\Pi},\left[\vec{H}, X_{\Lambda}\right]\right)+\sigma\left(X_{\Pi},\left[\vec{H}, X_{\Pi}\right]\right) \\
= & \sigma\left(\left[\vec{H}, X_{\Lambda}\right], X_{\Lambda}\right)+\sigma\left(X_{\Pi},\left[\vec{H}, X_{\Pi}\right]\right) \\
= & \dot{\Lambda}_{z}(t)\left(X_{\Lambda}\right)-\dot{\Pi}_{z}(t)\left(X_{\Pi}\right)>0 .
\end{aligned}
$$

From this we get that $z_{\infty}$ is a hyperbolic fixed point of $\phi^{t}$; we get by Hadamard-Perron theorem [8] that there exists a smooth submanifold in $T^{*} M$, which we will denote by $W_{\text {loc }}^{s}\left(z_{\infty}\right)$ and which is called the local stable manifold at $z_{\infty}$, such that

- $T_{z_{\infty}} W_{\mathrm{loc}}^{s}\left(z_{\infty}\right)=E_{z_{\infty}}^{-}$;

- there is a neighbourhood $U$ of $z_{\infty}$ such that

$$
W_{\mathrm{loc}}^{s}\left(z_{\infty}\right)=\left\{y \in U: d\left(\phi^{t}(y), z_{\infty}\right) \rightarrow 0 \text { as } t \rightarrow+\infty\right\} .
$$

Moreover, we can define the global stable manifold

$$
W^{s}\left(z_{\infty}\right)=\bigcup_{t>0} \phi^{-t}\left(W_{\mathrm{loc}}^{s}\left(\phi^{t}\left(z_{\infty}\right)\right)\right),
$$

which is a smooth manifold characterized by

$$
W^{s}\left(z_{\infty}\right)=\left\{y: d\left(\phi^{t}(y), z_{\infty}\right) \rightarrow 0 \text { as } t \rightarrow+\infty\right\} .
$$

\section{The Results}

Now we are ready to state the result:

Theorem 3.1. Let $M$ be a simply connected smooth manifold, and let $\varphi: T M \rightarrow \mathbb{R}$ be a smooth function that satisfies hypotheses (H1)-(H3). Let $\left\{\Lambda_{z}\right\}_{z}=\left\{T_{z}\left(T_{\pi(z)}^{*} M\right)\right\}_{z}$ and $\left\{\Pi_{z}\right\}_{z}, z \in M$, be two Lagrangian distributions that provide a splitting of $T\left(T^{*} M\right)$; assume that the generalized curvature of $\vec{H}$ w.r.t. the splitting is negative definite for any $z$. Then the problem (2.1) with final point $q_{\infty}$ admits a smooth optimal synthesis on $M$.

Proof. We already know that any optimal trajectory shall satisfy PMP, and, since the Hamiltonian is smooth, we also know that any smooth solution of the Hamiltonian system (2.5) in fact satisfies PMP, and vice versa. The following theorem (see [3]) provides a sufficient condition for an admissible trajectory to be optimal:

Theorem 3.2. Let $\mathcal{L}_{0}$ be a Lagrangian submanifold of $T^{*} M$, and call, for any $t, \mathcal{L}_{t}=\mathrm{e}^{t \vec{H}}\left(\mathcal{L}_{0}\right)$. Assume that for any $t \in[0, T]$ the restriction $\left.\pi\right|_{\mathcal{L}_{t}}$ of the projection $\pi: T^{*} M \rightarrow M$ is a diffeomorphism. Then for any $\lambda_{0} \in \mathcal{L}_{0}$ the normal extremal trajectory

$$
\tilde{q}(t)=\pi \circ \mathrm{e}^{t \vec{H}}\left(\lambda_{0}\right), \quad 0 \leq t \leq T
$$

realizes a strict minimum of the cost functional $\int_{0}^{T} \varphi(q(t), u(t)) \mathrm{d} t$ among all admissible trajectories $q(t), 0 \leq$ $t \leq T$, of system (2.1) with the same boundary conditions

$$
q(0)=\tilde{q}(0), \quad q(T)=\tilde{q}(T)
$$

This result is stated for finite final time $T$, but the generalization for infinite horizon problems is straightforward. 
We are going to apply Theorem 3.2 to the global stable manifold $W^{s}\left(z_{\infty}\right)$, thus proving that all the optimal trajectories for our problem are projections of stable solutions to (2.5). Then, the optimal synthesis is thus constructed: we put for any $q \in M$

$$
X(q):=\pi_{*}(\vec{H}(\lambda)),
$$

with $\lambda \in W^{s}\left(z_{\infty}\right)$ and $\pi(\lambda)=q$. This vector field is smooth, because the Hamiltonian is smooth and $W^{s}\left(z_{\infty}\right)$ projects diffeomorphically on $M$; since the optimal trajectories are projections of the integral curves of $\vec{H}$, they are actually integral curves of $X$.

The proof that $W^{s}\left(z_{\infty}\right)$ satisfies the hypotheses of Theorem 3.2 is split into two steps: in the first one we prove the Lagrangianity of the stable manifold, and that the restriction $\left.\pi\right|_{W^{s}\left(z_{\infty}\right)}$ is a local diffeomorphism and a proper mapping of $W^{s}\left(z_{\infty}\right)$ on its image (note in fact that the stable manifold is $\phi^{t}$-invariant, so it is sufficient to prove the condition only for $\left.W^{s}\left(z_{\infty}\right)\right)$. In the second step, we will prove that actually $W^{s}\left(z_{\infty}\right)$ projects surjectively on $M$, hence getting the existence of a (global) optimal synthesis.

\subsection{Regularity of the projection}

Lemma 3.1. $W^{s}\left(z_{\infty}\right)$ is a Lagrangian submanifold of $T^{*} M$.

Proof. Let $X, Y \in T_{z} W^{s}\left(z_{\infty}\right), z \in W^{s}\left(z_{\infty}\right)$; then, since the Hamiltonian flow preserves the symplectic form, for any $t$

$$
\sigma(X, Y)=\sigma\left(D_{z} \mathrm{e}^{t \vec{H}} X, D_{z} \mathrm{e}^{t \vec{H}} Y\right) \rightarrow 0 \quad \text { as } \quad t \rightarrow+\infty .
$$

Lemma 3.2. The restriction of the projection $\left.\pi\right|_{W^{s}\left(z_{\infty}\right)}$ is a covering of its image.

Proof. $\left.\pi\right|_{W^{s}\left(z_{\infty}\right)}$ is clearly a smooth map, since the stable manifold is smooth.

$\left.\pi\right|_{W^{s}\left(z_{\infty}\right)}$ is also an immersion, and, in particular, a local diffeomorphism; in fact, let us assume that there is a vector $X \in \operatorname{ker} \pi \cap T_{z} W^{s}\left(z_{\infty}\right)$; we get that $\mathcal{Q}_{0}(X)=0$, since the projection of $X$ on the horizontal space vanishes. Since $Q_{t}(X) \rightarrow 0$ as $t \rightarrow+\infty$ and $\mathcal{L}_{\vec{H}} \mathcal{Q}$ is positive definite, we have that $\mathcal{Q}_{t}(X)=0$ for any $t \geq 0$, which implies that $D_{z} \phi^{t} X$ is vertical (i.e. $\left.\left(D_{z} \phi^{t} X\right)_{q}=0\right)$ for nonnegative $t$; since $\left[\vec{H}\left(\phi^{t}(z)\right), D_{z} \phi^{t} X\right] \notin T_{\phi^{t}(z)}\left(T_{\pi\left(\phi^{t}(z)\right)}^{*} M\right)$, we get a contradiction.

Let us now prove that $\left.\pi\right|_{W^{s}\left(z_{\infty}\right)}$ is a proper mapping: first of all, let $d(\cdot, \cdot)$ be the distance induced on $T^{*} M$ by the scalar product previously defined, and let $B_{r}(z)$ denote the ball of radius $r$ centered at $z$, for some $r>0, z \in T^{*} M$. Let $K$ be a compact set in $\pi\left(W^{s}\left(z_{\infty}\right)\right)$, and $\left\{z_{i}\right\}_{i}$ a sequence in $\left.\pi\right|_{W^{s}\left(z_{\infty}\right)} ^{-1}(K)$. The sequence is bounded: in fact, let us write the $z_{i}$ 's in coordinates, $z_{i}=\left(p_{i}, q_{i}\right)$ for any $i ; q_{i} \in K$ for any $i$, and hence they are bounded; the $p_{i}$ shall be bounded as well, because the stable manifold lies in the level $H^{-1}(0)$, and the Hamiltonian grows to $+\infty$ when $|p| \rightarrow+\infty$. Then the sequence $\left\{z_{i}\right\}_{i}$ converges, up to a subsequence, to some $\bar{z} \in \pi^{-1}(K)$; let us assume that $\bar{z} \notin W^{s}\left(z_{\infty}\right)$. Let us consider the Hamiltonian trajectories whose initial conditions are given by these $z_{i_{k}}$ 's: by continuity, for any small $\varepsilon>0$ and any $T>0$ we can find $k$ such that $\phi^{t}\left(z_{i_{k}}\right) \in B_{\varepsilon}\left(\phi^{t}(\bar{z})\right)$ for any $0 \leq t \leq T$. Since we assumed that $\bar{z} \notin W^{s}\left(z_{\infty}\right)$, $\phi^{t}(\bar{z})$ shall go to infinity (see Cor. 10), which means that for any $\rho>0$ we can always find a $t^{\prime} \geq T$ such that $\left.\phi^{t^{\prime}}(\bar{z}) \notin B_{2 \rho}\left(z_{\infty}\right)\right)$.

Now recall that $z_{i_{k}} \in W^{s}\left(z_{\infty}\right)$, which means that $\phi^{t}\left(z_{i_{k}}\right)$ reaches $z_{\infty}$ with exponential rate, that is there are two positive constants $b, \gamma$ such that $\left\|\vec{H}\left(\phi^{t}\left(z_{i_{k}}\right)\right)\right\| \leq b \mathrm{e}^{-\gamma t}\left\|\vec{H}\left(z_{i_{k}}\right)\right\|$. So we have that

$$
\int_{t}^{+\infty}\left\|\vec{H}\left(\phi^{\tau}\left(z_{i_{k}}\right)\right)\right\| \mathrm{d} \tau \leq b\left\|\vec{H}\left(z_{i_{k}}\right)\right\| \int_{t}^{+\infty} \mathrm{e}^{-\gamma \tau} \mathrm{d} \tau=b\left\|\vec{H}\left(z_{i_{k}}\right)\right\| \frac{\mathrm{e}^{-\gamma t}}{\gamma} .
$$

We can chose $\varepsilon$ so small and $\rho$, T so large that $b\left\|\vec{H}\left(z_{i_{k}}\right)\right\| \frac{\mathrm{e}^{-\gamma T}}{\gamma}<\rho$, which means that the trajectory $\phi^{t}\left(z_{i_{k}}\right)$ cannot reach $z_{\infty}$. This is a contradiction; hence $\bar{z}$ belongs to the stable manifold, and hence $\left.\pi\right|_{W^{s}\left(z_{\infty}\right)} ^{-1}(K)$ is compact.

Since $\left.\pi\right|_{W^{s}\left(z_{\infty}\right)}$ is a proper local diffeomorphism, it is also a smooth covering (of its image); if its image is simply connected, we can conclude that the map $\left.\pi\right|_{W^{s}\left(z_{\infty}\right)}$ is also a global diffeomorphism (onto its image). 


\subsection{Existence of the optimal synthesis}

Now we will prove that the projection $\left.\pi\right|_{W^{s}\left(z_{\infty}\right)}$ is onto, in order to get the global version of Theorem 3.2 and the existence of the optimal synthesis.

Let us concentrate on the set $\pi\left(W^{s}\left(z_{\infty}\right)\right)$; it is an open subset of $M$, since it is the image of a local diffeomorphism.

Moreover, it is closed in $M$ too: indeed, we shown that the stable manifold $W^{s}\left(z_{\infty}\right)$ is closed. Since the restriction $\left.\pi\right|_{W^{s}\left(z_{\infty}\right)}$ is a proper map (and in particular a closed map), $\pi\left(W^{s}\left(z_{\infty}\right)\right)$ shall be closed in $M$.

Then, we conclude that $\pi\left(W^{s}\left(z_{\infty}\right)\right)=M$.

Remark 3.1. It is crucial to assume that the function $u \mapsto \varphi(q, u)$ has superlinear growth. In fact, assume by contradiction that there exists a direction $u_{j}$ on which the function $\varphi$ has linear growth for large $\|u\|$, which implies that $\partial_{u_{j}} \varphi$ tends to a constant as $\|u\|$ goes to infinity. Then there exists an $M>0$ such that $\sup _{u}\langle\lambda, u\rangle-\varphi(q, u)=+\infty$ if the $j$ th component of $\lambda$ exceeds $M$. If the maximized Hamiltonian is not defined, the flow itself is not globally defined.

\section{The Euclidean Case}

Theorem 4.1. Consider the problem

$$
\min _{q(t)} \int_{0}^{\infty} \varphi(q(t), u(t)) \mathrm{d} t
$$

with

$$
\begin{array}{ll}
\dot{q}=u & q(0)=q_{0} \\
& q(t) \rightarrow q_{\infty} \text { as } t \rightarrow+\infty \quad q \in \mathbb{R}^{n},
\end{array}
$$

where $\varphi$ is smooth and strongly convex in the pair $(q, u)$ and the function $u \mapsto \varphi(q, u)$ has superlinear growth for any $q$, and there is a point $q_{\infty}$ such that $\varphi\left(q_{\infty}, 0\right)=0$ and $\frac{\partial \varphi}{\partial q}\left(q_{\infty}, 0\right)=0$.

Then there exists a hyperbolic fixed point $z_{\infty}$ with $\pi\left(z_{\infty}\right)=q_{\infty}$ of the Hamiltonian system associated to (4.1), and the problem (4.1) with final point $q_{\infty}=\pi\left(z_{\infty}\right)$ admits a smooth optimal synthesis on $\mathbb{R}^{n}$.

Proof. As above, PMP let us associate to problem (4.1) a Hamiltonian $h_{u}(p, q)=\langle p, q\rangle-\varphi(p, q)$, and its maximized $H(p, q)=\max _{u} h_{u}(p, q)$.

The hypotheses on $\varphi$ imply that the maximized Hamiltonian $H(p, q)$ is smooth and that the assumption (H3) is satisfied.

Let us now choose, for any $(p, q), \Lambda_{(p, q)}=\operatorname{span}\left\{\partial_{p_{1}}, \ldots, \partial_{p_{n}}\right\}(p, q)$ and $\Pi_{(p, q)}=\operatorname{span}\left\{\partial_{q_{1}}, \ldots, \partial_{q_{n}}\right\}(p, q)$. We have that $\dot{\Lambda}_{(p, q)}>0$ and $\dot{\Pi}_{(p, q)}<0$; in fact, let $X=\sum_{i=1}^{n} X_{i} \partial_{p_{i}} \in \Lambda_{(p, q)}$ and $Y=\sum_{i=1}^{n} Y_{i} \partial_{q_{i}} \in \Pi_{(p, q)}$; then

$$
\begin{gathered}
\dot{\Lambda}_{(p, q)}(X)=\sigma([\vec{H}, X], X)=\sum_{i=1}^{n} X_{i} X_{j} \frac{\partial^{2} H}{\partial p_{i} \partial p_{j}}, \\
\dot{\Pi}_{(p, q)}(Y)=\sigma([\vec{H}, Y], Y)=\sum_{i=1}^{n} Y_{i} Y_{j} \frac{\partial^{2} H}{\partial q_{i} \partial q_{j}}
\end{gathered}
$$

by direct computation, we get that

$$
\frac{\partial^{2} H}{\partial p_{i} \partial p_{j}}=\left.\sum_{l, m} \frac{\partial \bar{u}_{l}}{\partial p_{i}} \frac{\partial^{2} \varphi}{\partial u_{l} \partial u_{m}}\right|_{u=\bar{u}} \frac{\partial \bar{u}_{m}}{\partial p_{j}}
$$


which is positive definite. On the other hand,

$$
\begin{aligned}
\frac{\partial^{2} H}{\partial q_{i} \partial q_{j}} & =\frac{\partial^{2} h_{u}}{\partial q_{i} \partial q_{j}}-\left.\sum_{l, m} \frac{\partial \bar{u}_{l}}{\partial q_{i}} \frac{\partial^{2} h_{u}}{\partial u_{l} \partial u_{m}}\right|_{u=\bar{u}} \frac{\partial \bar{u}_{m}}{\partial q_{j}} \\
& =-\frac{\partial^{2} \varphi}{\partial q_{i} \partial q_{j}}+\sum_{l, m} \frac{\partial^{2} \varphi}{\partial q_{j} \partial u_{l}}\left(\frac{\partial^{2} \varphi}{\partial u_{l} \partial u_{m}}\right)^{-1} \frac{\partial \varphi}{\partial q_{i} \partial u_{m}}
\end{aligned}
$$

in fact, let us define the function $F: \mathbb{R}^{3 n} \rightarrow \mathbb{R}$ by $F(p, q, u)=p-\frac{\partial \varphi}{\partial u}(q, u)$; $\operatorname{since} \operatorname{rk}\left(J_{u} F\right)=n$, we can apply the Implicit Function Theorem and express locally the function $\bar{u}$ such that $\left.\frac{\partial \varphi}{\partial u}\right|_{u=\bar{u}}=0$ as a function of $p$ and $q$, and moreover we have that

$$
J \bar{u}=-\left(J_{u} F\right)^{-1}\left(J_{(p, q)} F\right)=\left(\frac{\partial^{2} \varphi}{\partial u^{2}}\right)^{-1}\left(\mathbb{I},-\frac{\partial^{2} \varphi}{\partial q \partial u}\right) .
$$

Let us perform a change of variable and assume that, in the point where we are computing the derivatives, the Hessian of $\varphi$ w.r.t. $u$ is the unit matrix, thus reducing (4.3) to

$$
\begin{aligned}
\frac{\partial^{2} H}{\partial q_{i}^{2}} & =-\frac{\partial^{2} \varphi}{\partial q_{i}^{2}}+\sum_{l=1}^{n}\left(\frac{\partial u_{l}}{\partial q_{i}}\right)^{2} \\
& =-\operatorname{Hess}\left(\left.\varphi\right|_{Q=\text { const }}\right)
\end{aligned}
$$

with $Q=\left(q_{1}, \ldots, \hat{q}_{i}, \ldots, q_{n}\right)$; hence $\frac{\partial^{2} H}{\partial q_{i}^{2}}<0$. Since we can perform any linear change of variable in the space of the $q$ 's obtaining the same expression (4.3), we can conclude that the second derivative of $H$ w.r.t. any direction in the space of coordinates is negative, i.e. $H$ is strictly concave w.r.t., hence $\operatorname{Hess}_{q}(H)$ is a negative-definite matrix.

This fact implies that the generalized curvature with respect to this splitting is negative definite; then we are under assumptions of Theorem 3.1.

Remark 4.1. The following example shows that the convexity of $\varphi(q, u)$ is not sufficient to assure that the generalized curvature w.r.t. the canonical splitting is negative definite.

In fact, let

$$
\varphi(q, u)=f(u)+U(q), \quad q, u \in \mathbb{R},
$$

with $f^{\prime \prime}(u)>0$ and $U^{\prime \prime}(q)>0$.

The generalized curvature is

$$
\begin{aligned}
R^{H}(p, q)= & \frac{1}{2}\left(\frac{\partial^{2} H}{\partial p^{2}}\right)^{-1}\left(\frac{\partial H}{\partial q}\right)^{2} \frac{\partial^{4} H}{\partial p^{4}}-\frac{1}{2}\left(\frac{\partial^{2} H}{\partial p^{2}}\right)^{-1} \frac{\partial^{2} H}{\partial q^{2}} \frac{\partial^{3} H}{\partial p^{3}} \frac{\partial H}{\partial p} \\
& +\frac{\partial^{2} H}{\partial p^{2}} \frac{\partial^{2} H}{\partial q^{2}}-\frac{3}{4}\left(\frac{\partial^{2} H}{\partial p^{2}}\right)^{-2}\left(\frac{\partial H}{\partial q}\right)^{2}\left(\frac{\partial^{3} H}{\partial p^{3}}\right) \\
= & \frac{1}{4 f^{\prime \prime}(\bar{u})^{4}}\left(3 U^{\prime}(q)^{2} f^{(3)}(\bar{u})^{2}-4 f^{\prime \prime}(\bar{u})^{3} U^{\prime \prime}(q)-2 \bar{u} f^{\prime \prime}(\bar{u})^{2} U^{\prime \prime}(q) f^{(3)}(\bar{u})\right. \\
& \left.-2 U^{\prime}(q)^{2} f^{(4)}(\bar{u}) f^{\prime \prime}(\bar{u})\right)
\end{aligned}
$$

choosing $\varphi(q, u)=u^{4}+u^{2}+q^{2}$, we get

$$
R(\bar{u}, q)=\frac{-\left(1+6 \bar{u}^{2}\right)^{2}\left(1+12 \bar{u}^{2}\right)+q^{2}\left(72 \bar{u}^{2}-6\right)}{\left(1+6 \bar{u}^{2}\right)^{4}},
$$

which is not a negative function. 


\section{The 1-Dimensional CASE}

Let us now consider the optimal problem (4.1)-(4.2) with $q \in \mathbb{R}$; we will investigate whether the problem admits an optimal synthesis only looking at the phase portrait of the dynamical system generated by the maximized Hamiltonian $H(p, q)$.

We assume that $\varphi$ is smooth and strongly convex in the second variable, and that the maximized Hamiltonian $H(p, q)=\max _{\dot{q}} p \dot{q}-\varphi(q, \dot{q})$ is well defined for any $(p, q)$; these hypotheses imply that $H(p, q)$ is a smooth function strongly convex in $p$.

At a first moment, we do the following assumption:

(A1) for any $q \in \mathbb{R}$ the function $p \mapsto H(p, q)$ has a (unique, by convexity) minimum;

this implies that there is a smooth curve $\gamma$ that divides $\mathbb{R}^{2}$ into two disjoint regions $\Gamma^{+}$and $\Gamma^{-}$such that $\frac{\partial H}{\partial p}>0$ for any $(p, q) \in \Gamma^{+}, \frac{\partial H}{\partial p}<0$ for any $(p, q) \in \Gamma^{-}$, and $\frac{\partial H}{\partial p}=0$ if $(p, q) \in \gamma$; (A1) implies that $\gamma$ is projected surjectively onto the horizontal axis. By strict convexity of $H$ w.r.t. $p, \gamma$ is never tangent to vertical lines.

We remark that hypothesis (A1) is automatically satisfied if we require $\varphi$ to have superlinear growth w.r.t. $u$.

All equilibrium points of $\vec{H}$ belong to $\gamma$ and, if we denote by $\vec{H}_{\text {hor }}$ the component of $\vec{H}$ along the horizontal direction, $\vec{H}_{\text {hor }}$ points in the positive directions for $(p, q) \in \Gamma^{+}$, in the negative direction if $(p, q) \in \Gamma^{-}$, and it vanishes on $\gamma$.

Let us now classify the possible phase portraits of such a Hamiltonian. We always assume that the equilibrium points are nondegenerate; if they are isolated degenerate, then we can always put things in general position provoking local differences in the phase diagram, while the global behaviour does not change much.

1 equilibrium point. Since the Hamiltonian flow preserves volumes, the equilibrium point can only be a center or a saddle; sinks, sources, stable and unstable nodes, stable and unstable foci cannot arise.

If the equilibrium point is a center, then there is no orbit reaching it in an infinite time, hence the problem (4.1) has never solution.

Let us now suppose that the equilibrium point is a saddle. First of all, we notice that, since the function $p \mapsto H(p, q)$ is strongly convex for any $q$, for any fixed $q$ there are at most two distinct values $p_{1}, p_{2}$ such that $\left(p_{i}, q\right)$ belongs to the same level of $H$ for $i=1,2$. This implies that the semi-trajectories belonging to the stable and the unstable manifolds are unbounded: in fact, by Poincaré-Bendixon theorem, if a semitrajectory is bounded, either it arises from another critical point, either its $\alpha$-limit set is a periodic trajectory. Neither of these cases can occur; the first one because by hypothesis there is no other equilibrium points; the second one cannot occur since, by convexity of $H$ w.r.t. $p$, each stable semi-trajectory cannot wind around the equilibrium point, otherwise we would have more than two points with the same horizontal coordinate belonging to the same level of $H$.

We can also prove that the stable and the unstable semi-trajectories are projected bijectively onto the horizontal axis. If the projection of a semi-trajectory that belongs to the stable or the unstable manifold is bounded, then the horizontal velocity along it vanishes while the trajectory goes to infinity, that contradicts convexity of $H$ w.r.t. $p$; this implies surjectivity. Injectivity is a consequence of the fact that there can exist at most two points with the same horizontal coordinate that belong to the same sublevel of the Hamiltonian, and that both the stable and the unstable trajectory are projected onto the horizontal axis.

Then, the infinite-horizon problem admits a solution for any initial point $q_{0}$.

2 equilibrium points. As we saw, equilibrium points for this Hamiltonian can only assume the shape of saddles and centers, so phase portraits for two equilibria can be obtained combining these possibilities. Call the two points $z_{1}$ and $z_{2}$, with $\pi\left(z_{1}\right)<\pi\left(z_{2}\right)$.

2 saddles. This combination is forbidden due to convexity of $H$ w.r.t $p$, for this implies the non-existence of vertical trajectories and because of the fact that $\vec{H}_{\text {hor }}$ has positive verse on $\Gamma^{+}$and negative verse on $\Gamma^{-}$. As shown in Figures 3 and 4, this gives rise to trajectories that intersect each other (in particular, in Fig. 3 it is shown the situation in which the two equilibria belong to the same sublevel, in Fig. 4 the one in which they 


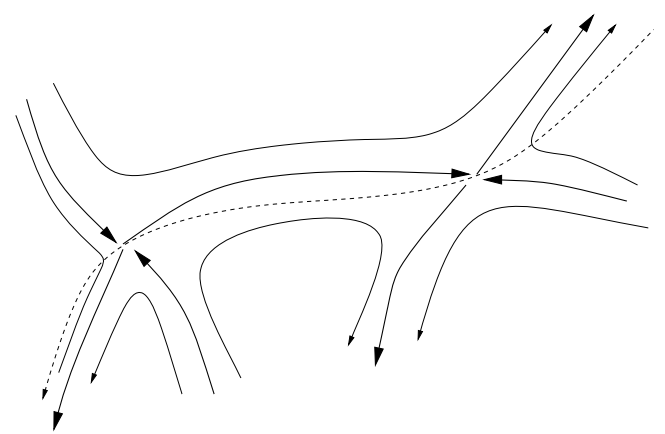

Figure 3. Two equilibria: saddle-saddle (1) (forbidden case).

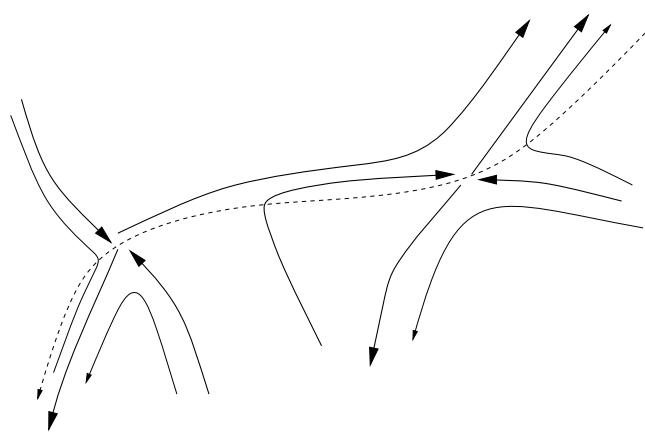

Figure 4. Two equilibria: saddle-saddle (2) (forbidden case).

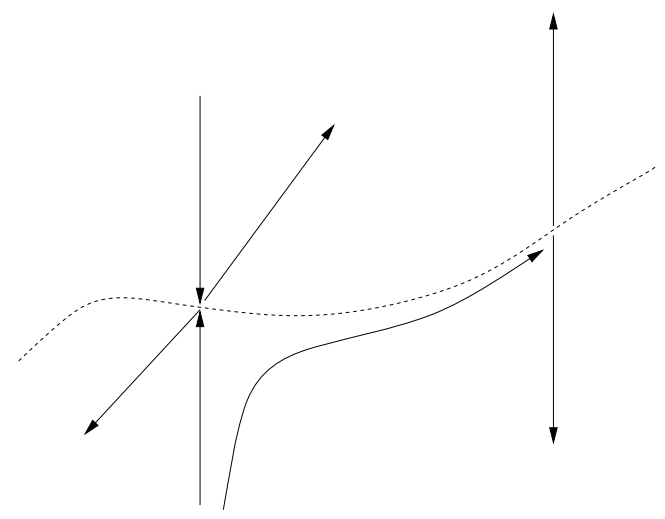

Figure 5. Two equilibria: saddle-saddle (3) (forbidden case).
Figure 6. Two equilibria: center-center (4) (forbidden case).

belong to different sublevels). In Figure 5 it is illustrated that, if we try to avoid these intersections, we would get trajectories whose horizontal velocity has wrong verse.

2 centers. This combination is again forbidden; in fact, by the structure of the Hamiltonian, the closed trajectories around each of the equilibria are covered in the same verse (clockwise). This fact leads up to a contradiction; if, as in Figure 6, the closed orbits get nearer, then there shall be a line (called $h$ in Fig. 6) that separates the region of orbits around $z_{1}$ from the region of orbits around $z_{2}$ and, on this line, the component of $\vec{H}$ parallel to it shall vanish; but since this line shall cross $\gamma$ and it's transversal to it, then there shall be a point on which $\vec{H}$ vanishes, which is in contradiction with the fact that $H$ has only two critical points.

Otherwise, if there is family of closed trajectories that surround both critical points, then, getting nearer $z_{1}$ and $z_{2}$, there shall be at least another (or a continuum of) equilibrium point, as it is shown in Figure 9.

1 centre and 1 saddle. In this case, there can be two kind of phase diagram; let $z_{1}$ be the saddle point and $z_{2}$ the centre. In the first case, shown in Figure 7, one unstable semitrajectory and one stable semitrajectory of $z_{1}$ act together as a separatrix between the closed orbits around $z_{2}$ and the unbounded orbits of the other part of the plane. Repeating previous arguments, we see that these trajectories are diffeomorphically projected onto the horizontal line, and then we get the existence of minima of problem (4.1) with $q_{\infty}=\pi\left(z_{1}\right)$ for any $q_{0} \in \mathbb{R}^{n}$. 


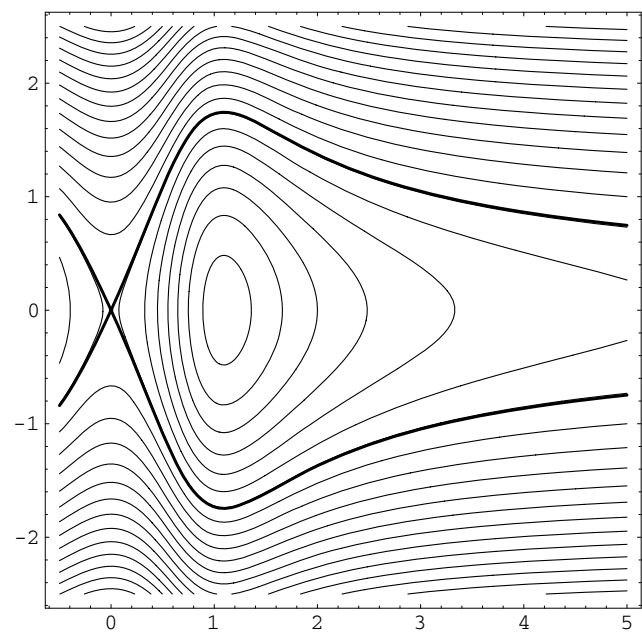

Figure 7. Two equilibria: saddle-center (1) (allowed).

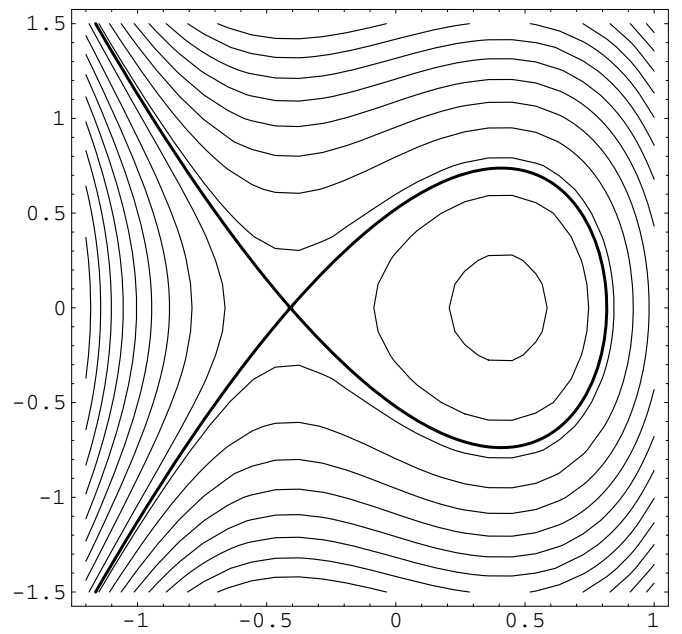

Figure 8. Two equilibria: saddle-center (2) (allowed).

A sample Hamiltonian with this behaviour is the function $H(p, q)=\frac{1}{2} p^{2}+U(q)$, where $U(q)$ behaves as $-q^{2}$ for $q<\pi\left(z_{1}\right)$ and in a neighbourhood of $\pi\left(z_{1}\right)$, has a local minimum in $\pi\left(z_{2}\right)$, and then grows monotonically and assumes values strictly less than $U\left(\pi\left(z_{1}\right)\right)$.

In the second case, illustrated in Figure 8, a stable and an unstable semitrajectory of $z_{1}$ join together in a closed separatrix $\sigma$; in the closed region surrounded by $\sigma$ there are the closed orbits around $z_{2}$, in the outer there are open orbits. In this case, there is a $\hat{q}$ such that the infinite-horizon problem has solution only for initial point $q_{0} \leq \hat{q}$ if $\pi\left(z_{2}\right)>\pi\left(z_{1}\right)$, and for $q_{0} \geq \hat{q}$ if $\pi\left(z_{2}\right)<\pi\left(z_{1}\right)$.

A sample Hamiltonian in this case is the function $H(p, q)=\frac{1}{2} p^{2}+q^{3}-\alpha q, \alpha>0$.

3 equilibrium points. As noticed all critical points lie on $\gamma$, and, moreover, we know from previous arguments that some configurations (two saddles or two centers side by side) are forbidden; hence, we can only find two situations: saddle-centre-saddle and centre-saddle-centre. Let us call $z_{1}, z_{2}$ and $z_{3}$ the equilibria in such a way that $\pi\left(z_{1}\right)<\pi\left(z_{2}\right)<\pi\left(z_{3}\right)$.

Centre-saddle-centre. Let $z_{1}$ and $z_{3}$ be the two centers. There is a closed trajectory $\sigma$ that surrounds both the centers and that passes through the saddle point that is a separatrix between the closed orbits around $z_{1}$ or $z_{3}$ and the outer orbits (that, depending on the problem, can be open or closed). In this case, the infinite-horizon problem with final point $\pi\left(z_{2}\right)$ has solution only for $q_{0} \in \pi(\sigma)$, while there is no Hamiltonian trajectories which reach a point $z$ such that $\pi(z)=q_{1}$ or $\pi(z)=q_{3}$ in an infinite time, thus the infinite-time problem with these conditions has no solution.

This behaviour can be generated by a Hamiltonian like $H(p, q)=\frac{1}{2} p^{2}+q^{4}+\alpha q^{3}-\beta q^{2}, \beta>0$.

Saddle-centre-saddle. We have to distinguish two cases: in the first one, shown in Figure 10, the two saddle points $\left(z_{1}\right.$ and $\left.z_{3}\right)$ belong to the same sublevel of $H$; in the other one, shown in Figure 11 , they belong to different sublevels.

Let us consider the former case; we notice that one stable semitrajectory arising from $z_{1}$ joins with one unstable semitrajectory of $z_{2}$, and vice versa; these two trajectories form a separatrix between the closed orbits around the centre and the unbounded orbits. Obviously, there is no solution for the infinite-horizon problem with final point $\pi\left(z_{2}\right)$. Let us consider the problem with final point $\pi\left(z_{1}\right)$ : for initial time $q_{0} \leq \pi\left(z_{1}\right)$ the infinite-horizon solution always exists, since the unstable manifold is diffeomorphically projected onto the halfline $\left\{q \leq \pi\left(z_{1}\right)\right\}$; for $\pi\left(z_{1}\right) \leq q_{0}<\pi\left(z_{2}\right)$, the problem has again solution, since a point on the separatrix reaches $\pi\left(z_{1}\right)$ in an infinite time; for $q_{0} \geq \pi\left(z_{2}\right)$, there are no Hamiltonian trajectories reaching a point $z \in \mathbb{R}^{2}$ such that $\pi(z)=\pi\left(z_{1}\right)$ in infinite time, hence there is no solution. We can repeat the same argument to say that 


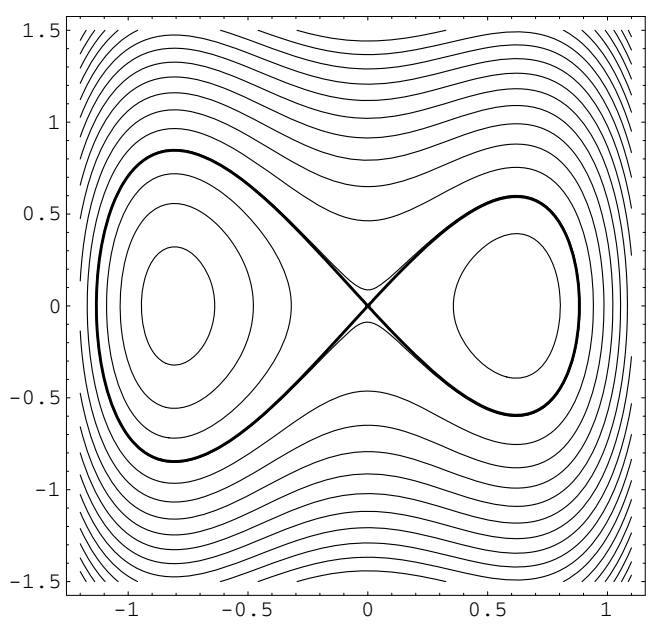

Figure 9. Three equilibria: center-saddle-center.

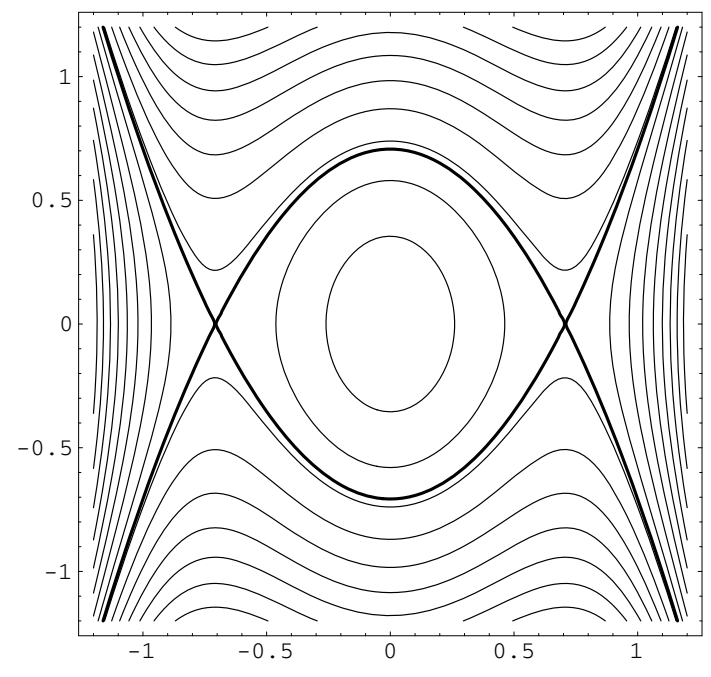

Figure 10. Three equilibria: saddle-center-saddle (1).

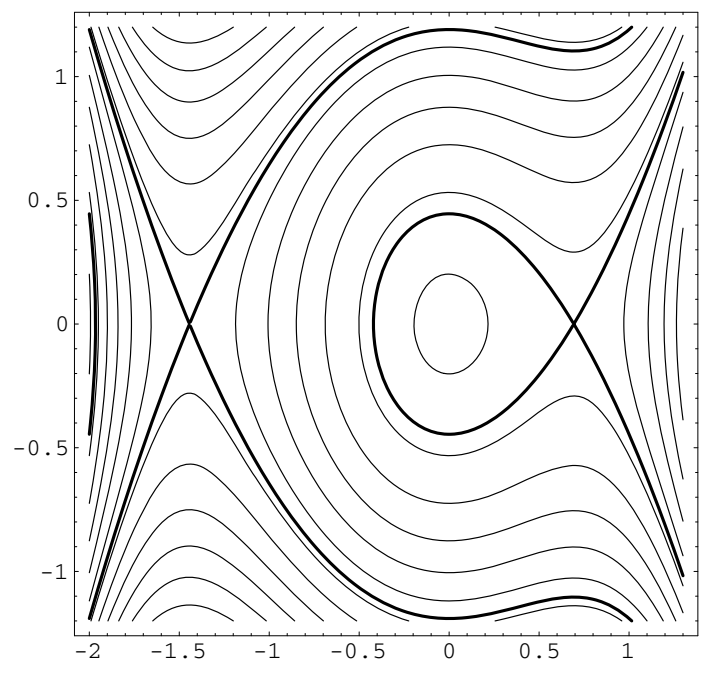

Figure 11. Three equilibria: saddle-center-saddle (2).

the infinite-horizon problem for final point $\pi\left(z_{2}\right)$ admits solution for $q_{0} \geq \pi\left(z_{2}\right)$ and for $\pi\left(z_{1}\right)<q_{0} \leq \pi\left(z_{2}\right)$ (on the separatrix).

A sample Hamiltonian that has this phase diagram is $H(p, q)=\frac{1}{2} p^{2}-q^{4}+\beta q^{2}, \beta>0$.

Let us now focus on the second case; about the problem with final state $\pi\left(z_{3}\right)$, we can repeat the same arguments used in the situation depicted in Figure 7: there is a $\hat{q}$ such that the infinite-horizon problem admits solution if and only if $q \geq \hat{q}$; otherwise, problem (4.1) with final state $\pi\left(z_{1}\right)$ has a solution for any $q_{0} \in \mathbb{R}$; as seen, there is no solution for $q_{\infty}=\pi\left(z_{2}\right)$.

This phase diagram can arise with a sample Hamiltonian as $H(p, q)=\frac{1}{2} p^{2}-q^{4}-\alpha q^{3}+\beta q^{2}, \alpha \neq 0, \beta>0$.

More than 3 equilibrium points. We get from previous arguments that all the equilibrium points lie on $\gamma$ and they can only be aligned alternating saddle points and centers. Then all the possible configurations can be deduced by previous results. 


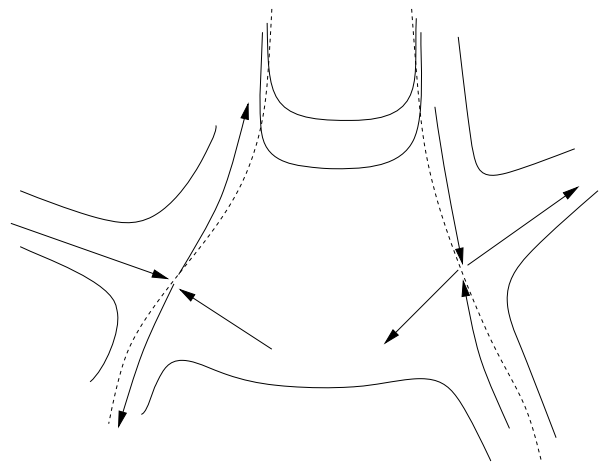

FiguRE 12. Two saddles: allowed if we remove hypothesis (A1).

Let us now see what happens if we remove hypothesis (A1). Since we ask that $\vec{H}$ has at least one equilibrium point, there shall exist at least one $\bar{q}$ for which the function $p \mapsto H(p, \bar{q})$ has a minimum; then, there exists a curve $\gamma$ that divides $\mathbb{R}^{2}$ into two regions as above, and such that $\gamma$ passes through the minimum of $H(\cdot, \bar{q})$. What we cannot say is that $\gamma$ is projected onto the horizontal axis: it can happen that its projection is bounded. This implies that we cannot guarantee that the stable manifold is projected onto the horizontal line, hence losing the existence of the optimal synthesis (but we still have local existence of minimizers).

Moreover, we notice that there can be cases in which there exist two (or more) disjoint curves $\gamma_{i}$ that divides the regions where $\frac{\partial H}{\partial p}>0$ from the ones where $\frac{\partial H}{\partial p}<0$; in such cases, it remains true that on the same curve there cannot lie two saddle points or two centers side by side, but we can have situations such as the one depicted in Figure 12 in which the two critical points are saddle points.

For such cases, we just repeat that our analysis is only local and permits to prove the existence of minimizers only for some initial points $q_{0}$.

Acknowledgements. The authors would like to thank Piotr Przytycki for useful suggestions.

\section{REFERENCES}

[1] A.A. Agrachev, Geometry of Optimal Control Problem and Hamiltonian Systems, in Nonlinear and Optimal Control Theory, Lecture Notes in Mathematics 1932, Fondazione C.I.M.E., Firenze, Springer-Verlag (2008).

[2] A.A. Agrachev and R.V. Gamkrelidze, Feedback-invariant optimal control theory and differential geometry, I. Regular extremals. J. Dyn. Contr. Syst. 3 (1997) 343-389.

[3] A.A. Agrachev and Yu.L. Sachkov, Control Theory from the Geometric Viewpoint. Springer-Verlag, Berlin (2004).

[4] A. Bressan and Y. Hong, Optimal control problems on stratified domains. Netw. Heterog. Media 2 (2007) 313-331.

[5] G.M. Buttazzo, M. Giaquinta and S. Hildebrandt, One-dimensional variational problems: an introduction. Oxford University Press (1998).

[6] L. Cesari, Optimization theory and applications. Springer-Verlag (1983).

[7] R.V. Gamkrelidze, Principles of Optimal Control Theory. Plenum Press, New York (1978).

[8] A. Katok and B. Hasselblatt, Introduction to Modern Theory of Dynamical Systems. Cambridge University Press, Cambridge (1995).

[9] A.V. Sarychev and D.F.M. Torres, Lipschitzian regularity of minimizers for optimal control problems with control-affine dynamics. Appl. Math. Optim. 41 (2000) 237-254.

[10] M.P. Wojtkovski, Magnetic flows and Gaussian thermostats on manifolds of negative curvature. Fund. Math. 163 (2000) 177-191. 